\title{
Degree and location of metacarpal angulations
}

\author{
Laxminarayan Bhandari, Cynthia Sathekga, Francisco Aguilar, Luis Vicentela, Elkin Galvis \\ Christine M. Kleinert Institute for Hand and Microsurgery, Louisville, KY 40202, USA. \\ Correspondence to: Dr. Laxminarayan Bhandari, Christine M. Kleinert Institute for Hand and Microsurgery, 225 Abraham Flexner Way, Suite 850, \\ Louisville, KY 40202, USA. E-mail: lbhandari@cmki.org
}

How to cite this article: Bhandari L, Sathekga C, Aguilar F, Vicentela L, Galvis E. Degree and location of metacarpal angulations. Plast Aesthet Res 2017;4:174-7.

\section{Article history: \\ Received: 9 Sep 2017 \\ Accepted: 21 Sep 2017 \\ Published: 20 Oct 2017}

\section{Key words:}

Metacarpals,

plate fixation,

anatomy

\section{INTRODUCTION}

Metacarpal fractures are a common injury of the hand, with an incidence of $13.6 / 100,000$ in the United States each year ${ }^{[1]}$. The angulation and displacement are crucial factors in determining operative management. There are various options for fixation of metacarpal fractures which include $\mathrm{K}$ wires, intramedullary nails, plates and screws, and external fixators. Plate and screw fixation is commonly employed for complex injuries $^{[2]}$.

\begin{abstract}
Aim: Fractures of metacarpals are commonly encountered in hand surgery. For adequate fixation, a thorough knowledge about the anatomy is essential. While fixing the metacarpals with plates and screws, plates are bent to contour the dorsal surface. However, there are no reference values in literature for the location and degree of angulation. The authors studied the dorsal surface of metacarpals in cadavers to gather data regarding the location and degree of angulation of the dorsal cortex. Methods: Cadaveric dissections of 118 metacarpals from 30 hands were performed. A true lateral view of each metacarpal was taken using fluoroscopy. These pictures were analyzed using Image $\mathrm{J}$ software. The dorsal cortex angle was measured in each image, and the center of rotation of angulation (CORA) was identified. The distance from the CORA to the base of metacarpal was measured and calculated as a percentage of the metacarpal length. Results: The average dorsal angle of the metacarpals was $11.5^{\circ}$. The average angles for each metacarpal were as follows: 2 nd metacarpal $=13^{\circ}$ (range, 6-26 $6^{\circ}$; $\mathrm{SD}, 4.73), 3 \mathrm{rd}=10^{\circ}\left(\right.$ range, $\left.1-25^{\circ} ; \mathrm{SD}, 5.28\right), 4 \mathrm{th}=11^{\circ}\left(\right.$ range, $\left.1-20^{\circ} ; \mathrm{SD}, 4.45\right), 5$ th $=12^{\circ}$ (range, $2-24^{\circ} ; \mathrm{SD}, 5.11$ ). The average location of the CORA from the base of the metacarpal as a percentage of the metacarpal length was identified as follows: $53.5 \%$ for the index finger, $52.1 \%$ for the long finger, $48.3 \%$ for the ring finger and $50.3 \%$ for the small finger. Conclusion: These measurements are able to serve as reference values for plate bending while operating on a metacarpal fracture or metacarpal corrective osteotomy.
\end{abstract}

During plate fixation, it is a common practice to bend the plates ${ }^{[3]}$. If the plate is not adequately bent, tightening the screws will result in a gap in the opposite cortex [Figure 1]. The location and degree of plate bending can be challenging to predict, especially in cases of a comminuted fracture, or fracture with bone loss. Therefore, a thorough knowledge of the anatomy of the dorsal surface is important. However, literature is scarce with this regard. The various metacarpal parameters described in literature include capital and subcapital angulations, proximal articular inclination,

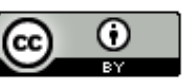

This is an open access article licensed under the terms of Creative Commons Attribution 4.0 International License (https://creativecommons.org/licenses/by/4.0/), which permits unrestricted use, distribution, and reproduction in any medium, as long as the original author is credited and the new creations are licensed under the identical terms.

For reprints contact: service@oaepublish.com

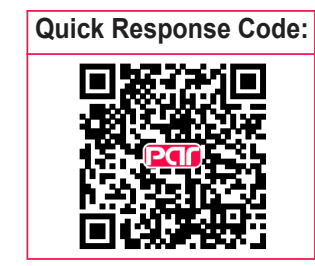



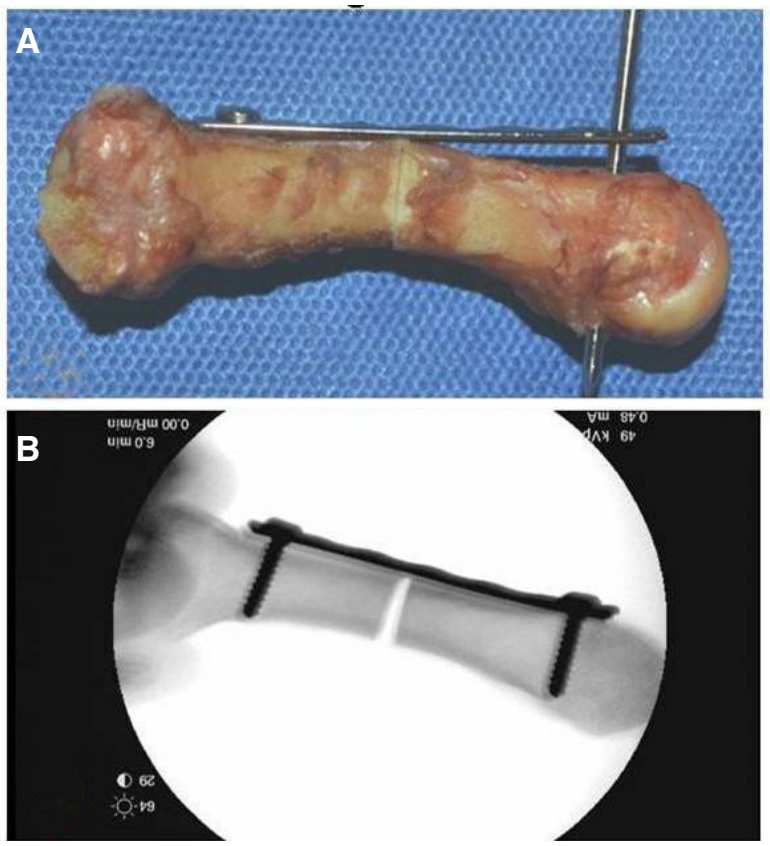

Figure 1: A: metacarpal with a plate placed across a transverse fracture; $\mathrm{B}$ : tightening the screws without bending the plate results in gap formation

and the shaft bending angles ${ }^{[4,5]}$. These measurements do not aid in plate bending.

It is technically difficult to obtain the dorsal angulations on a conventional lateral radiograph in a living patient due to overlap of metacarpals. Thus anatomical study is necessary.

\section{METHODS}

The study was performed in cadaveric metacarpal models. Statistical power studies demonstrated that 100 specimens were required to obtain statistical significance. After obtaining approval from the Human Tissue Committee, thirty cadaveric hands were dissected to harvest 118 metacarpal bones. Two hands had one digit missing. The bones were dissected free from the surrounding soft tissue.

After harvesting the metacarpal, a true lateral view of each metacarpal was taken using fluoroscopy. The pictures were analysed by Image $\mathrm{J}$ software (National Institute of Health, Bethesda, Maryland, USA).

\section{Dorsal cortex angle and centre of rotation of angulation}

Using the Image $\mathrm{J}$ software on the lateral view, two lines were drawn. The first line was drawn from the base of the metacarpal, along the dorsal surface distally. A second line was drawn from the neck of the metacarpal, along the dorsal surface proximally. The point where these two lines meet was identified as the center of rotation of angulation (CORA). The angle between the two lines was recorded as the dorsal cortex angle [Figure 2].

\section{Location of CORA-CORA ratio}

The location of the CORA was calculated as a ratio as follows: the distance from the CORA to the metacarpal base was measured against the total length of the metacarpal to get the CORA ratio [Figure 2].

The metacarpals were assigned numbers, and measurements were taken by blinding the observer to avoid observer expectancy bias.

\section{RESULTS}

\section{Dorsal cortical angle}

Table 1 demonstrates the mean dorsal cortical angles and standard deviations in the 30 cadaveric hands. The average angles were as follows: 2nd metacarpal $=13^{\circ}$ (range, $6-26^{\circ} ; \mathrm{SD}, 4.73$ ), $3 \mathrm{rd}=10^{\circ}$ (range, $1-25^{\circ} ; \mathrm{SD}$ $5.28), 4$ th $=11^{\circ}\left(\right.$ range, $\left.1-20^{\circ} ; \mathrm{SD}, 4.45\right), 5$ th $=12^{\circ}$
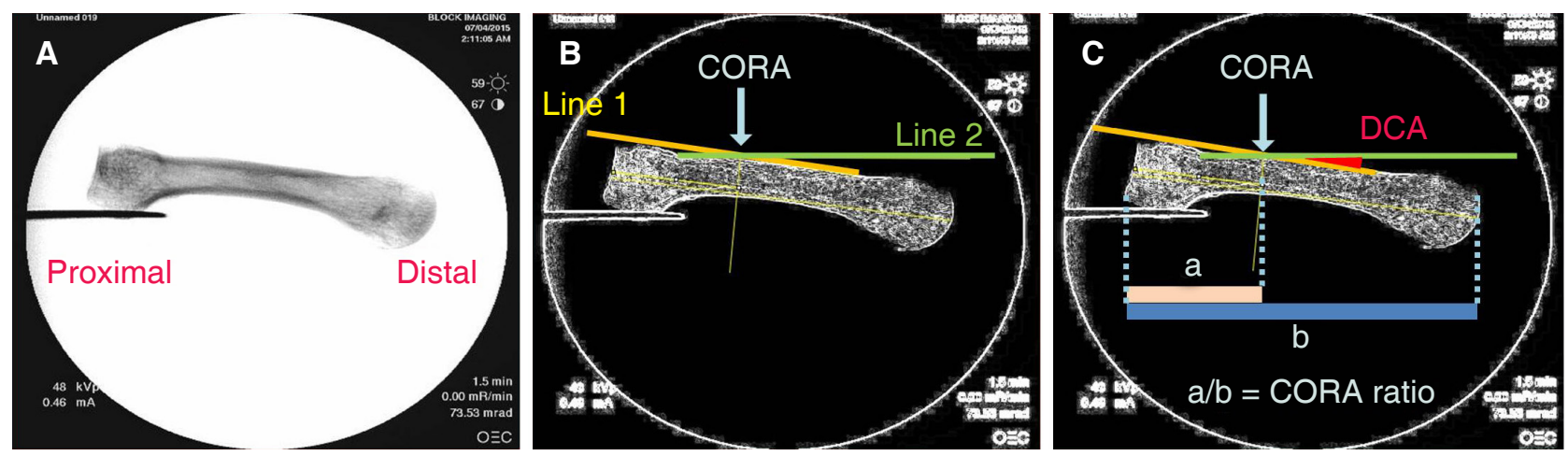

Figure 2: Showing the calculation of DCA and location of CORA. A: lateral fluoroscopy view of metacarpal; B: two lines were drawn. The first line was drawn from the base of the metacarpal, along the dorsal surface distally. The second line was drawn from the neck of the metacarpal, along the dorsal surface proximally. The point where these two lines meet was identified as the CORA. The angle between the two lines was taken as the DCA; C: the distance from the CORA to the metacarpal base was measured against the total length of the metacarpal to get the CORA ratio; DCA: dorsal cortical angle; CORA: center of rotation of angulation 
Table 1: Dorsal cortical angle

\begin{tabular}{lccc}
\hline & Mean $\left({ }^{\circ}\right)$ & Range $\left(^{\circ}\right)$ & Standard deviations \\
\hline 2nd & 13 & $6-26$ & 4.73 \\
3rd & 10 & $1-25$ & 5.28 \\
4th & 11 & $1-20$ & 4.45 \\
5th & 12 & $2-24$ & 5.11 \\
\hline
\end{tabular}

(range, $2-24^{\circ}$; SD, 5.11). There was no progressive difference as we moved from the II digit to the $\mathrm{V}$ digit.

\section{CORA ratio}

The following CORA ratios were obtained: $53.5 \%$ for the index finger (range, $42-85 \%$; SD, 12.5 ), $52.1 \%$ for the long finger (range, $32-100 \%$; SD, 17.8), 48.3 for the ring finger (range, $34-86 \%$; SD, 11.5 ) and $50.3 \%$ for the small finger (range, $27-86 \%$; SD, 12.4) [Table 2]. The location of CORA was found to be consistent across the four digits [Figure 3].

\section{DISCUSSION}

The knowledge of anatomical characteristics of metacarpals is crucial in diagnosis of metacarpal fractures and planning treatment. Assessment of the metacarpals is routinely done by radiographs. Braakman ${ }^{[4]}$ defined various radio graphical parameters of metacarpals of the ring and small fingers. The purpose was to gain consistency in reporting and to reduce inter-observer variations. These parameters include 4 axes and 4 angles. The axes were: (1) full-shaft axis: line through the centers of the shaft, measured at $1 / 3$ and $2 / 3$ of the bone length; (2) capital axis: line drawn perpendicular to a line through the widest part of the caput, indicating the end of the cartilage cap; (3) sub capital axis: the "logic" axis through the middle of the subcapital neck; and (4) proximal articular line: the tangent to the 2

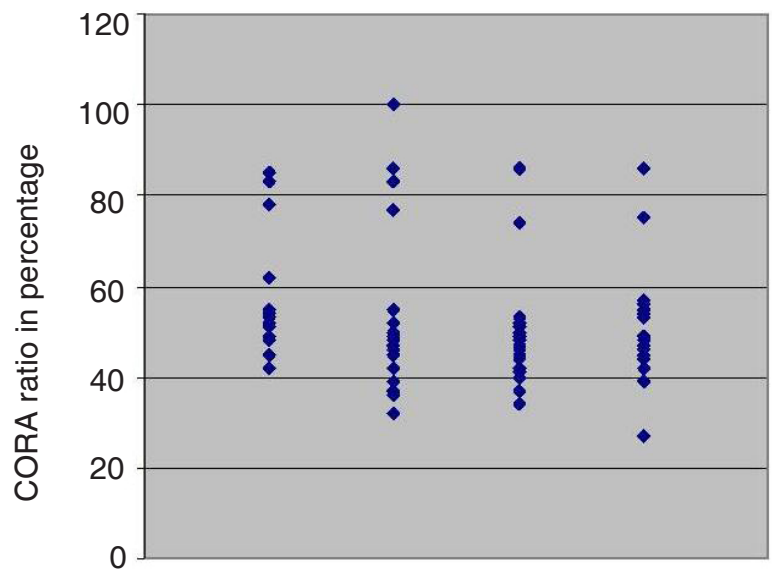

Metacarpals 2nd 3rd 4th 5th

Figure 3: Scatter diagram showing distribution of CORA ratio. CORA: center of rotation of angulation
Table 2: Center of rotation of angulation

\begin{tabular}{lccc}
\hline & Mean (\%) & Range (\%) & Standard deviations \\
\hline 2nd & 53.5 & $42-85$ & 12.5 \\
3rd & 52.1 & $32-100$ & 17.8 \\
4th & 48.3 & $34-86$ & 11.5 \\
5th & 50.3 & $27-86$ & 12.4 \\
\hline
\end{tabular}

bony eminences in case of a concave bony ending, as a line through the points indicating the widest osseous space in case of a convex cortex ending, and in case of a flat bony ending as a line parallel to this surface. The angles were: (1) capital axis angle (CAA): angle between the capital-axis and the full-shaft axis; (2) sub CAA: this is formed by the angle between the subcapital axis and the full-shaft axis; (3) shaft bending axis (SBA): this lies between the proximal and distal shaft-axis. The proximal and distal shaftaxis are defined by the 2 lines connecting the centers of the proximal shaft (measured as $1 / 3$ and 1/2 of the metacarpal length) and the distal shaft (measured as $1 / 2$ and $2 / 3$ ); and (4) the proximal articular angle: this lies between the full-shaft axis and the proximal articular line.

In their study, Braakman ${ }^{[4]}$ reported CAA of $22^{\circ}$ and $27^{\circ}$ on the postero-anterior view and $19^{\circ}$ and $24^{\circ}$ on the oblique views for the ring and small metacarpals, respectively. They found that the SBA was $2^{\circ}$ in all tested digits in both projections. Rivlin et al. ${ }^{[5]}$ analysed the CAA and SBA using computer tomography with three dimensional reconstruction. They showed that CAA averaged $14^{\circ}$ and $12^{\circ}$ in the ring and small metacarpals, respectively. Apex dorsal SBA averaged $12^{\circ}$ and $10^{\circ}$ in the ring and small metacarpals, respectively. Although these studies describe important anatomical landmarks which aid in repairing the fracture, they do not provide any detail regarding the "surgically important" dorsal surface.

In our study, we specifically measured the dorsal surface angulations. Since plate fixation involves the dorsal surface, the knowledge of its anatomy is imperative for successful plating. It has been traditionally considered that the dorsal surface is convex ${ }^{[6]}$. However, our results suggest that it is more angular than it is curved. In our study we obtained the average CORA ratio and dorsal cortex angle, which answers "where" and "how much" the angulation is. The numbers that we developed can serve as reference values for future metacarpal fixation procedures.

Knowledge of these angulations would be even more important in surgical correction of malunions. Birndorf et al..$^{[]}$ studied the effect of angulation on metacarpal fractures and found that with progressive increase in metacarpal 
angulation, there was an increase in tendon excursion, tendon load, and work required for flexion. Thus, returning the metacarpal to normal alignment is imperative.

In any curved long bone, the concave surface bears the compressive force. When such bones are fractured, the load bearing osteosynthesis should ideally tackle this concave surface. In the context of metacarpals, the volar surface would be the compression cortex. However, the easily accessible dorsal surface is preferred for plating, even though it is not the compression cortex. Metacarpals have attachments of $\mathrm{A} 1$ pulleys, and the flexor tendons, passing under these A1 pulleys, exert a flexion force on the distal part of the metacarpal. In setting metacarpal fractures, the flexor tendons can lead to volar angulation of the distal fragment. This may also account for increased incidence of screw loosening seen in the distal fragment ${ }^{[8]}$. Since the angle of the metacarpals is in the same direction as the flexion force, it may contribute to volar displacement as well as screw loosening.

In conclusion, the dorsal surface of metacarpals has an angulation rather than a curvature. The location of the angulation is somewhat consistent at the middle of the metacarpal. The degree of angulation is variable.

\section{DECLARATIONS}

\section{Acknowledgments}

The authors wish to thank the individuals of the University of Louisville Willed Body Program who donated their bodies and tissues for the advancement of education and research.

\section{Authors' contributions}

Analysis and interpretation of data, drafting of manuscript and critical revision: L. Bhandari
Study conception and design; acquisition, analysis and interpretation of data: C. Sathega, F. Aguilar, L. Vicentela, E. Galvis

Drafting of manuscript and critical revision: E. Galvis

\section{Financial support and sponsorship}

None.

\section{Conflicts of interest}

There are no conflicts of interest.

\section{Patient consent \\ Not applicable.}

\section{Ethics approval}

This study obtains approval from the Human Tissue Committee.

\section{REFERENCES}

1. Nakashian MN, Pointer L, Owens BD, Wolf JM. Incidence of metacarpal fractures in the US population. Hand (N Y) 2012;7:426-30.

2. Firoozbakhsh KK, Moneim MS, Howey T, Castaneda E, Pirela-Cruz MA. Comparative fatigue strengths and stabilities of metacarpal internal fixation techniques. J Hand Surg Am 1993;18:1059-68.

3. Fricker R, Kastelec M, Nuñez F, Axelrod T. Metacarpals. Available from: https://www2.aofoundation.org/wps/portal/surgery?showPage= diagnosis\&bone $=$ Hand\&segment $=$ Metacarpals. [Last accessed on 12 Oct 2017]

4. Braakman M. Normal radiographic angulation in the 4th and 5th metacarpal: a reference guide. Eur J Radiol 1996;22:38-41.

5. Rivlin M, Kim N, Lutsky KF, Beredjiklian PK. Measurement of the radiographic anatomy of the small and ring metacarpals using computerized tomographic scans. Hand (N Y) 2015;10:756-61.

6. Kamath JB, Harshvardhan, Naik DM, Bansal A. Current concepts in managing fractures of metacarpal and phalangess. Indian J Plast Surg 2011;44:203-11.

7. Birndorf MS, Daley R, Greenwald DP. Metacarpal fracture angulation decreases flexor mechanical efficiency in human hands. Plast Reconstr Surg 1997;99:1079-83; discussion 1084-5.

8. Kollitz KM, Hammert WC, Vedder NB, Huang JI. Metacarpal fractures: treatment and complications. Hand (N Y) 2014;9:16-23. 